\section{Fate of Nanoplastics in polar waters: Experimental evidence for expulsion from sea ice to saltwater}

\author{
ALICE PRADEL ${ }^{1}$, MAUD GAUTIER ${ }^{2}$, CHRISTIAN LE \\ CARLIER DE VESLUD ${ }^{2}$, DOMINIQUE BAVAY ${ }^{2}$ AND \\ JULIEN GIGAULT ${ }^{3}$ \\ ${ }^{1}$ CNRS UMR 6118 \\ ${ }^{2}$ Université Rennes 1, CNRS, Géosciences Rennes \\ ${ }^{3}$ University of Laval \\ Presenting Author: alice.pradel@gmail.com
}

Polar ecosystems play key roles in many Earth-system processes, such as the redistribution of particulate material throughout the water column and across ocean surfaces during the freeze-thaw cycles of sea ice. It has become clear that this particulate material now inevitably includes microplastics $(1 \mu \mathrm{m}$ $5 \mathrm{~mm})$ and nanoplastics $(<1 \mu \mathrm{m})$ [1]. Indeed, during their long journey from continental source to the poles, plastic waste is degraded to particulate sizes. Once in polar seas, this degradation is enhanced by strong UV radiation. Microplastic concentrations in the Arctic ocean range from $10^{2}$ to $10^{3}$ particles per $\mathrm{m} 3$ and are expected to rise [2]. In particular, the Barents and Greenland seas will form zones of plastic accumulation[3]. Strikingly, microplastic concentrations in sea ice are consistently higher, by a factor approximately 100 , compared to seawater[4].

However, while nanoplastics could represent an important proportion of the ocean's total plastic budget, their fate in polar waters remains unknown. This study provides the first mechanistic description of nanoplastic transfer between saltwater and ice. A freezing reactor was conceived which progressively and partially froze microplastic and nanoplastic dispersions, after which their affinity for ice and liquid was quantified. The particles used had different degrees of environmental relevance: spherical polystyrene (PS) with diameters $200 \mathrm{~nm}$ (nPSL-200), $350 \mathrm{~nm}$ (nPSL-350), PS particles with irregular shapes (nPS-360) as well as PS microplastics with sizes 150 to $400 \mu \mathrm{m}(\mu \mathrm{PS})$. Contrary to microplastics, nanoplastics are strongly expulsed from ice, as seen in Figure 1. Concentrations of nanoplastics in ice is heterogenous, with accumulation zones in brine pockets. So, sea ice creates a medium-term sink for microplastics, but it accelerates nanoplastics' journey down the water column. Nanoplastics have a different behavior than microplastics due to their colloidal properties. This highlights the fact that nanoplastics cannot be lumped into the same category as microplastics.

[1] Bank, M. S. \& Hansson, S. V. (2019) Environ.Sci.Technol. 53, 13, 7177-7179

[2] Tekman, M. B. et al. (2020) Environ.Sci.Technol. 54, 4079-4090.

[3] van Sebille, E., et al. (2012) Environ.Res.Lett. 7, 044040.

[4] Peeken, I. et al. (2018) Nature Communications 9.

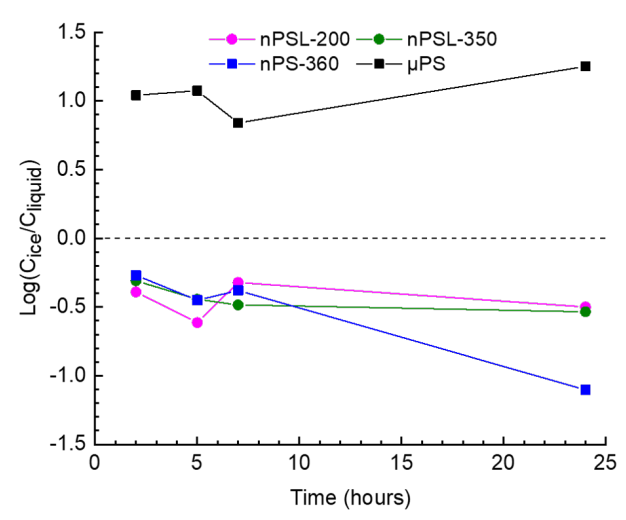

Figure 1: Logarithm of partition coefficient between ice and liquid phase $\left(C_{i c e} / C_{\text {liquid }}\right)$ of nanoplastics and microplastics. 